1 Universidade Estadual do Sudoeste da Bahia (Uesb) - Jequié - (BA), Brasil. icaro.ribeiro@ymail.com

2 Universidade Estadual do Sudoeste da Bahia (Uesb) - Jequié - (BA), Brasil.

rboery@gmail.com

3 Universidade Estadual do Sudoeste da Bahia (Uesb) - Jequié - (BA), Brasil. cacasotti@uesb.edu.br

4 Universidade Estadual do Sudoeste da Bahia (Uesb) - Jequié - (BA), Brasil. vidal.ivna@gmail.com

5 Universidade Estadual do Sudoeste da Bahia (Uesb) - Jequié - (BA), Brasil.

eboery@ig.com.br

\section{Qualidade de Vida de hipertensos atendidos na Atenção Primária à Saúde}

\author{
Quality of life of people with high blood pressure at the Primary \\ Health Care
}

Ícaro José Santos Ribeiro', Rita Narriman de Oliveira Boery², Cezar Augusto Casottiª Ivna Vidal Freire, $\mathbf{4}$ Eduardo Nagib Boery ${ }^{\mathbf{5}}$

RESUMO Objetivou-se analisar a Qualidade de Vida de hipertensos em Jequié-BA, e identificar possíveis fatores depressores. Trata-se de um estudo de corte transversal no qual aplicaram-se dois questionários e examinou-se cada indivíduo. A média da Qualidade de Vida geral foi de 67,5 [IC: 64.21-70.78]. O domínio manifestações psicológicas apresentou média de 88.01 [IC: 86.57-89.44] e o domínio manifestações somáticas 91.84 [IC: 90.76 - 92.91]. A Qualidade de Vida dos usuários acometidos por hipertensão arterial foi satisfatória. Quanto aos fatores depressores da Qualidade de Vida identificou-se que a escolaridade e a raça/cor dos entrevistados influenciam de forma acentuada nesta quantificação.

PALAVRAS-CHAVE Hipertensão; Qualidade de vida; Atenção Primária à Saúde.

ABSTRACT This study aimed to analyze the quality of life of hypertensive patients in Jequié-BA, and identify possible factors depressants. This is a cross-sectional study in which were applied two questionnaires and examined each individual. The overall quality of life mean was 67.5 [CI: 64.21-70.78]. The psychological manifestations domain showed an average of 88.01 [CI: 86.5789.44] and the somatic manifestations domain average 91.84 [CI: 90.76-92.91]. The Quality of Life of users affected by hypertension was satisfactory. Regarding factors that influence quality of life it was identified that education and race/color of the respondents influence in this quantification.

KEYWORDS Hypertension; Quality of life; Primary Health Care. 


\section{Introdução}

O modelo brasileiro de atenção à saúde originou-se de um processo de descentralização e regionalização, que entre outras medidas, implementou o Programa Saúde da Família (PSF). A atuação das equipes multiprofissionais em áreas adstritas, na assistência, prevenção e promoção da saúde aproximou a população dos serviços. Entre os programas englobados pelo PSF, o Hiperdia destaca-se, por atender usuários acometidos por duas das Doenças Crônicas Não Transmissíveis (DCNT) mais prevalentes, a hipertensão e o diabetes, que pelo caráter crônico, podem afetar a qualidade e o estilo de vida destes usuários.

A Hipertensão Arterial Sistêmica (HAS) é a DCNT de maior relevância clínica e a maior causadora de eventos cardíacos agudos, como o Infarto Agudo do Miocárdio (IAM). A prevalência global estimada de HAS em 2000 era de $26 \%$ e em 2025 poderia aumentar em $24 \%$ nos países desenvolvidos e $80 \%$ em países em desenvolvimento (KEARNEY ET AL., 2005). No Brasil, em 2010, a prevalência auto referida de HAS, na população adulta, variou de 13,8 a $29,2 \%$ (BRASIL, 2011).

O estilo de vida de pessoas acometidas por HAS deve ser alvo de modificações de caráter não apenas curativo, mas principalmente, preventivo. Desta forma, mudanças de estilo de vida potencialmente controlam os níveis da Pressão Arterial (PA) podendo diminuir a mortalidade cardiovascular. Entre as principais recomendações para prevenção e controle da HAS estão a alimentação saudável, consumo controlado de sódio e álcool, ingestão de potássio, combate ao sedentarismo e ao tabagismo (VI DBHA, 2010).

A HAS contribui significativamente para modificações na Qualidade de Vida (QV) das pessoas por interferir na capacidade física, emocional, interação social, atividade intelectual, exercício profissional e outras atividades do cotidiano (RABELO ET AL., 2011).

A QV é um elemento que tem sido alvo de crescente interesse científico e público em geral por ser um conceito inerentemente humano, relacionado ao grau de satisfação nas diferentes esferas (familiar, amorosa, social e ambiental). Compreende conhecimentos, experiências e valores de indivíduos e coletividades que se relacionam em diferentes momentos (MINAYO; HARTZ; BUSS, 2000).

Diante dessa problemática, este estudo objetivou analisar QV de usuários do programa Hiperdia de uma Unidade Básica de Saúde (UBS) do município de Jequié (BA), além de identificar fatores possivelmente depressores da QV.

\section{Material e método}

Estudo descritivo, de corte transversal, realizado em uma UBS localizada no bairro Jequiezinho-Jequié-BA, no período de janeiro a março de 2011.

Os participantes do estudo foram os usuários acometidos por HAS, cadastrados no programa Hiperdia, por meio de entrevistas semiestruturadas, realizada por pesquisador padronizado.

O cálculo amostral foi realizado com o programa Epi Info ${ }^{\mathrm{TM}}$ Versão 3.5.3 (2011), adotando a estimativa do fator QV de 69,7\% (MELCHIORS ET AL., 2010), erro de $5 \%$ e nível de confiança de $95 \%$. Sendo a população de hipertensos cadastrados na unidade de saúde da família de 203 indivíduos, a amostra necessária foi de 125 hipertensos.

Os indivíduos foram selecionados aleatoriamente, sendo entrevistados no dia agendado para consulta de enfermagem do Hiperdia. Após autorizar a participação e assinar o Termo de Consentimento Livre e Esclarecido (TCLE), aplicaram-se os questionários sócio-econômico-demográfico acrescido de questões referentes a hábitos de vida e o Minichal-Brasil (schulz ET AL., 2008), questionário de avaliação da QV devidamente validado e adaptado no Brasil, bem como realização de exame clínico.

Por meio do questionário sócio-econômico-demográfico e de hábitos de vida obtiveram-se as variáveis, gênero, idade em anos, 
escolaridade, raça/cor, uso de tabaco e álcool e prática de atividade física. Para a análise, estes dados foram categorizados, respectivamente, em feminino e masculino, adulto e idoso, sem e com escolaridade, branco e não branco. As questões referentes aos hábitos de vida foram dicotomizadas em sim ou não.

o Minichal-Brasil vem sendo utilizado para avaliar a QV e utiliza uma escala do tipo Likert variando de 0 a 3 pontos. Para o cálculo do somatório dos domínios, os escores foram convertidos para uma escala de 0 a 100, onde quanto mais próximo a 100 melhor a QV. O ponto de corte para a boa QV foram valores acima de 69,7 pontos (MELCHIORS ET AL., 2010).

As mensurações de peso (Kg) e estatura $(\mathrm{cm})$ foram obtidas por meio de balança mecânica com estadiômetro acoplado, devidamente calibrada, disponibilizada na UBS, sendo estas medidas empregadas para calculo do IMC por meio da fórmula 'peso/estatura ${ }^{2}$.

A Pressão Alterial (PA) foi aferida pelo método indireto com uso de aparelho de pressão oscilométrico automático (Omron ${ }^{\mathrm{TM}}$ ), em ambiente com temperatura controlada e de acordo com as VI Diretrizes Brasileiras de Hipertensão Arterial (VIDBHA, 2010) que recomenda entre outras orientações a medida da pressão arterial na posição sentada, com repouso prévio de 5 minutos em ambiente calmo.

Os participantes do estudo foram caracterizados em relação a aspectos sócio-econômicos-demográficos, modo de vida, por meio de frequências para dados categóricos e de médias, e desvios-padrão e medianas e intervalos para os dados contínuos com distribuição paramétrica e não paramétrica, respectivamente.

Utilizou-se o software estatístico Stata ${ }^{\top M}$, versão 12.0, na análise dos dados. O nível de significância adotado foi de $95 \%$. Por meio do teste Kolmogorov-Smirnov constatou-se a não normalidade da amostra, e desta forma os testes estatísticos não paramétricos foram empregados.

Estudo submetido e aprovado pelo Comitê de Ética em Pesquisa da Universidade Estadual do Sudoeste da Bahia (CEP/Uesb), Protocolo $\mathrm{n}^{\mathrm{o}} 135 / 2008$. Cumprindo as normas da Resolução 196/96 os indivíduos eram esclarecidos quanto aos aspectos da pesquisa e quando de acordo, autorizaram a coleta de dados, com a assinatura do Termo de Consentimento Livre e Esclarecido.

\section{Resultados}

Foram avaliados 125 usuários acometidos por HAS que passaram pela consulta de enfermagem do Hiperdia. A idade média foi de 60,39 anos ( $\mathrm{dp} \pm 13,24)$, com valores, máximo e mínimo, de 89 e 28 anos, $1^{\circ}$ e $3^{\circ}$ quartis de 52 e 70 anos. A tabela 1 apresenta os dados referentes ao perfil sócio-econômico-demográfico dos usuários acometidos por HAS participantes.

Tabela 1. Caracterização da amostra de indivíduos hipertensos estudada

\begin{tabular}{lrrr}
\hline Categoria & Variável & $\mathbf{n}$ & \% \\
\hline Sexo & Masculino & 37 & 29,6 \\
& Feminino & 88 & 70,4 \\
Escolaridade & Sem escolaridade & 34 & 27,2 \\
& Fundamental completo e/ou incompleto & 68 & 54,4 \\
Raça/cor & Médio completo e/ou incompleto & 23 & 18,4 \\
& Branco & 40 & 32,0 \\
& Preto & 39 & 31,2 \\
& Pardo & 46 & 36,8
\end{tabular}

Fonte: Elaboração própria 
A partir dos dados exibidos na tabela 1 verificou-se que os usuários acometidos por HAS da UBS, que participaram do estudo, são, em sua maioria, mulheres (70,4\%), de baixa escolaridade e da cor parda (36,8\%). O gráfico 1 apresenta a distribuição do gênero por grupo etário.

Gráfico 1. Distribuição da idade de pacientes hipertensos de acordo com o sexo

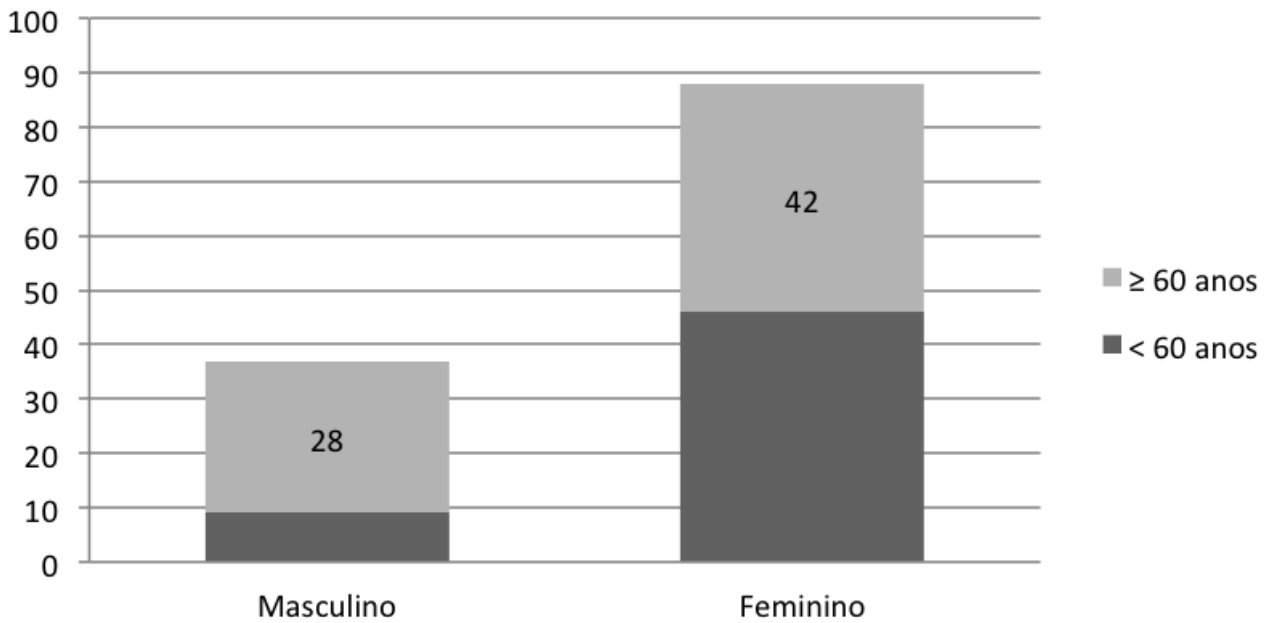

Fonte: Elaboração própria

Verificou-se diferença estatisticamente significante $(\mathrm{p}=0.006)$ entre as variáveis, idade e gênero. Vale ressaltar que $60 \%$ $(n=42)$ dos 70 usuários com idade acima de 60 anos de idade eram do gênero feminino.

A investigação dos hábitos de vida evidenciou que $93 \%$ (n=117) não faziam uso atual de cigarro, porém 38,4\% ( $\mathrm{n}=48)$ afirmaram já ter feito uso em algum momento da vida. A mesma abordagem em relação ao álcool mostrou que 84,8 \% não fazem uso atual, mas $52,8 \%$ ( $\mathrm{n}=66$ ) já o fizeram (gráfico 2).

Gráfico 2. Hábitos de vida dos indivíduos acometidos ou não por hipertensão

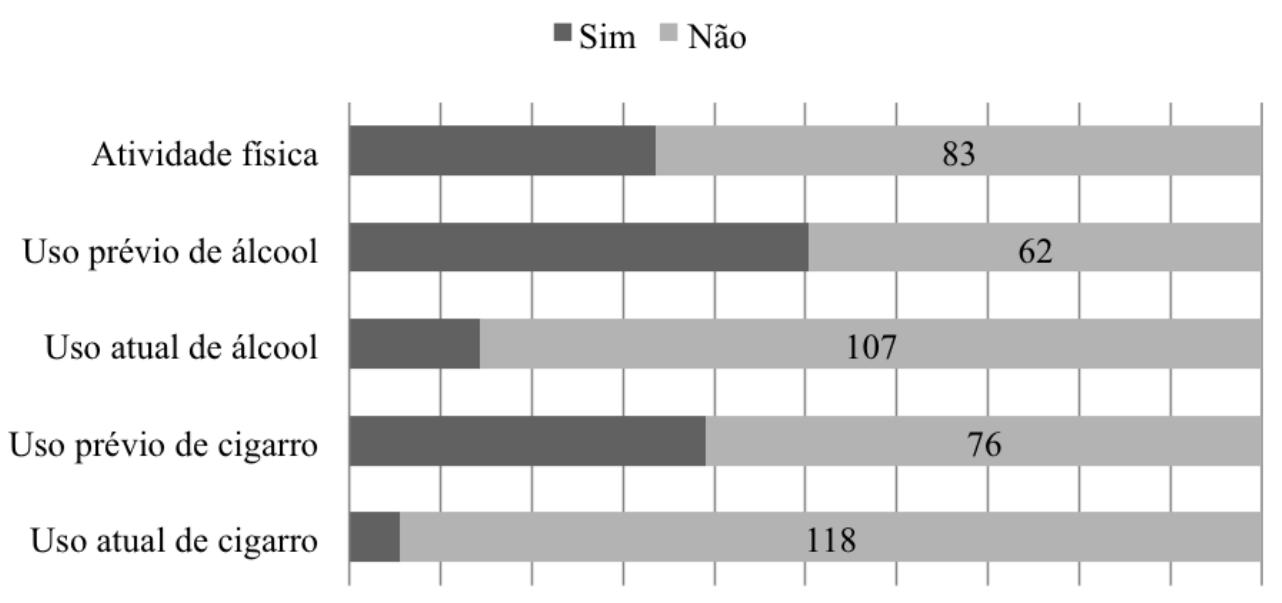


Conforme observado no gráfico 2, a prevalência do uso de tabaco, álcool, e de realização de prática de atividade física entre os hipertensos, acompanhados na UBS, foi respectivamente $39,2 \%, 50,4 \%$ e $33,6 \%$.

Foi identificada diferença estatisticamente significativa $(p<0.001)$ entre uso de álcool e tabaco de maneira conjugada. Ademais, identificou-se também, diferença estatisticamente significativa entre gênero e uso de tabaco $(p=0.002)$.

Quanto às medidas antropométricas, peso e altura, e o indicador antropométrico Índice de Massa Corporal (IMC), foi possível identificar que uma leve relação negativa ( $r=-$ 0.307) na qual o aumento da idade acarreta diminuição do IMC, a tabela 2 apresenta os resultados:

Tabela 2. Valores das médias das medidas antropométrica dos hipertensos assistidos pela Unidade Básica de Saúde

\begin{tabular}{lrrrrrrr}
\hline & $N$ & $\bar{\chi}(\mathrm{Kg})$ & $\mathrm{Dp}$ & $\bar{\chi}(\mathrm{cm})$ & $\mathrm{Dp}$ & $\bar{\chi} \mid \mathrm{MC}$ & $\mathrm{Dp}$ \\
\hline$>60$ anos & 55 & 72 & $\pm 14,77$ & 154 & \pm 8 & 30,05 & 5,38 \\
$\leq 60$ anos & 70 & 65 & $\pm 12,73$ & 155 & \pm 10 & 26,86 & 4,26 \\
\hline
\end{tabular}

Fonte: Elaboração própria

Em relação a PA, 64\% dos pacientes apresentaram valores considerados normais e $36 \%$ apresentaram elevada. A média da PA sistólica foi de $138 \mathrm{mmHg}(\mathrm{dp} \pm 25,5)$ e a diastólica de $83 \mathrm{mmHg}(\mathrm{dp} \pm 12,78)$. Os níveis pressóricos oscilaram entre o mínimo de 110x60 mmHg a um máximo de 220x120 $\mathrm{mmHg}$, mostrando que mesmo em tratamento medicamentoso os usuários apresentam picos hipertensivos.

Os resultados do Minichal-Brasil foram categorizados em dois domínios: manifestações psicológicas e somáticas. O domínio manifestações psicológicas apresentou média de 88.01 [CI: 86.57 - 89.44] (dp $\pm 8,27$ ), com valor máximo e mínimo de 100 e 56 , sendo o $1^{\circ}$ e $3^{\circ}$ quartis, 84 e 94 pontos. Já no domínio manifestações somáticas a média foi de 91.84 [CI: 90.76 - 92.91] (dp $\pm 6,94)$, com valor máximo e mínimo de 100 e 56 , sendo o $1^{\circ}$ e $3^{\circ}$ quartis 88 e 96 pontos.

Ao aplicar o teste de correlação de Spermann entre os domínios do Minichal (manifestações somáticas e o estado mental), foi possível evidenciar relação de força mediana e positiva $(\mathrm{r}=0.497)$.

Utilizando-se uma escala do tipo Likert, os entrevistados responderam a seguinte pergunta 'Quanto a HAS e o tratamento da mesma interferem na sua vida?'. Foi obtido o seguinte valor da média 1.976 ( $\mathrm{dp} \pm 0,94)$. Estes valores correspondem ao pior resultado da QV obtido neste estudo, uma vez que $67,2 \%(n=84)$ dos entrevistados responderam que a HAS interfere muito ou bastante em suas vidas.

Avaliando o escore total do MinichalBrasil (soma dos dois domínios e a questão única), e comparando aos resultados encontrados, foi possível identificar que a população apresenta boa QV com média geral de 67,5 [CI: 64.21-70.78]. Ao aplicar o teste de Wilcoxon verificou-se que não houve diferença estatisticamente significante na $\mathrm{QV}$ do grupo que apresentou pressão elevada e o de níveis pressóricos normais $(p>0,05)$.

Ainda buscando resultados relevantes à pesquisa optou-se por comparar as variáveis para mensurar o grau de dependência entre elas e a QV (tabela 3). 
Tabela 3. Associação entre as variáveis estudadas nos pacientes hipertensos assistidos pela Unidade Básica de Saúde, segundo a Qualidade de Vida

\begin{tabular}{|c|c|c|c|c|c|c|c|}
\hline \multirow{3}{*}{ Variável } & \multirow{3}{*}{ Categoria } & \multicolumn{4}{|c|}{ Qualidade de vida } & \multirow[t]{3}{*}{ Valor $\mathrm{p}$} & \multirow[t]{3}{*}{$\chi^{2 \star}$} \\
\hline & & \multicolumn{2}{|c|}{ Baixa } & \multicolumn{2}{|c|}{ Alta } & & \\
\hline & & $n$ & $\%$ & $n$ & $\%$ & & \\
\hline \multirow{2}{*}{ Sexo } & Masculino & 13 & 10,4 & 24 & 19,2 & \multirow{2}{*}{0.1954} & \multirow{2}{*}{1,68} \\
\hline & Feminino & 42 & 33,6 & 46 & 36,8 & & \\
\hline \multirow{2}{*}{ Raça/cor } & Brancos & 13 & 10,4 & 27 & 21,6 & \multirow{2}{*}{$<0.001$} & \multirow{2}{*}{13.18} \\
\hline & Não brancos & 57 & 45,6 & 28 & 22,4 & & \\
\hline \multirow{2}{*}{ Escolaridade } & Alfabetizado & 26 & 20,8 & 65 & 52 & \multirow{2}{*}{0.001} & \multirow{2}{*}{9.74} \\
\hline & Não alfabetizado & 20 & 16 & 14 & 11,2 & & \\
\hline \multirow{2}{*}{ Idade } & Adulto & 26 & 20,8 & 29 & 23,2 & \multirow{2}{*}{0.4151} & \multirow{2}{*}{0.66} \\
\hline & Idoso & 28 & 22,4 & 42 & 33,6 & & \\
\hline \multirow{2}{*}{ Uso de álcool } & Bebe & 32 & 25,6 & 31 & 24,8 & \multirow{2}{*}{0.1222} & \multirow{2}{*}{2.38} \\
\hline & Não bebe & 23 & 18,4 & 39 & 31,2 & & \\
\hline \multirow{2}{*}{ Uso de tabaco } & Fuma & 22 & 17,6 & 27 & 21,6 & \multirow{2}{*}{0.8709} & \multirow{2}{*}{0.03} \\
\hline & Não fuma & 33 & 26,4 & 43 & 34,4 & & \\
\hline \multirow{2}{*}{ Atividade física } & Sim & 15 & 12 & 27 & 21,6 & \multirow{2}{*}{0.1843} & \multirow{2}{*}{1.76} \\
\hline & Não & 40 & 32 & 43 & 34,4 & & \\
\hline \multirow{2}{*}{ Hipertensão } & Normal & 31 & 24,8 & 49 & 39,2 & \multirow{2}{*}{0.1805} & \multirow{2}{*}{1.79} \\
\hline & Elevada & 23 & 18,4 & 22 & 17,6 & & \\
\hline \multirow{2}{*}{ IMC } & Eutrófico & 14 & 11,2 & 17 & 13,6 & \multirow{2}{*}{0.8806} & \multirow{2}{*}{0.02} \\
\hline & Distrófico & 41 & 32,8 & 53 & 42,4 & & \\
\hline
\end{tabular}

Fonte: Elaboração própria

${ }^{*}$ Chi quadrado de Person

\section{Discussão}

O acometimento da QV mediante o aparecimento de diferentes patologias vem sendo investigado por diversos estudos (ALEXANDRE; CORDEIRO; RAMOS, 2008; BRITO ET AL., 2008; CORSO ET AL., 2009; LEMOS, 2008; RABELO ET AL., 2011). OS achados evidenciam que uma diminuição na QV está normalmente associada a casos mais graves das doenças e que a manutenção da boa QV é de grande relevância para que estas comorbidades não afetem sobremaneira a vida das pessoas acometidas por elas.

Todavia, a subjetividade do conceito e mensurações da QV são fatores potencialmente limitantes dos resultados obtidos. Também, a utilização de questionário validado diminui as discrepâncias metodológicas potencialmente existentes.
Neste estudo, assim como em outras pesquisas envolvendo hipertensos (BLOCH; MELO; NOGUEIRA, 2008; CIPULLO ET AL., 2010; COSTA ET AL., 2007; JESUS ET AL., 2008), identificou-se um maior percentual de mulheres acometidas por hipertensão (70,4\%). A média de idade dos entrevistados foi de 60,4 anos, sendo este um fator de risco e grande contribuinte para a epidemiologia da HAS, pois existe relação direta e linear entre a PA e idade (VIDBHA, 2010).

Alguns fatores biológicos intrínsecos influenciam na prevalência e na gravidade da hipertensão, sendo estas maiores nos negros (COSTA ET AL., 2007; LIMA ET AL., 2010; PASSOS; ASSIS; BARRETO, 2006; PESSUTO; CARVALHO, 1998), o que pode estar relacionado a fatores étnicos e/ou socioeconômicos. De forma semelhante ao apresentado pela literatura, o maior percentual de entrevistados foi da etnia 'não branca' (68\%). 
Entre as variáveis analisadas, o uso de álcool e tabaco foram os que apresentaram diferenças estatisticamente significativas $(\mathrm{p}<0.001)$, sendo que $28,8 \%(n=36)$ dos informantes fumam e bebem de forma associada. Associação semelhante entre fumo e consumo de álcool foi encontrado numa pesquisa realizada em São Paulo (SANTA-HELENA; NEMES; ELUF-NETO, 2010).

Foi possível efetuar o cálculo da prevalência do uso de tabaco na amostra estudada evidenciando que 5,6\% fumam e $39,2 \%$ já fumaram algum tempo em suas vidas. No Brasil, as diferenças metodológicas na abordagem ao uso do cigarro dificultam sobremaneira a comparação dos achados. Todavia, estudos que comparam apenas o status de fumante ou não, encontraram prevalências variando de $11,9 \%$ (MATOS; LADEIA, 2003) a 30,7\% (COSTA ET AL., 2007).

De forma semelhante, a prevalência do uso de álcool foi de $14,4 \%$ e $50,4 \%$ afirmaram que beberam no passado. No Brasil, o uso de álcool é prática bastante difundida. Estudos realizados apontaram o consumo de bebidas alcoólicas oscilando entre 23,7\% (HELENA; NEMES; ELUF-NETO, 2010) e 53,7\% (CONCEIÇÃO ET AL., 2006).

A prática de atividade física foi negada por $66,4 \%(n=83)$ dos informantes. Esse dado também foi constatado por estudo (OLIVEIRA; NOGUEIRA, 2010) no qual de 147 indivíduos, $106(72,1 \%)$ não praticam nenhum tipo de atividade física e num outro (SANTAHELENA; NEMES; ELUf-NETO, 2010) onde $71,1 \%$ dos hipertensos entrevistados não praticavam atividade física.

O Índice de Massa Corpórea dos informantes desta pesquisa apontou que $44 \%$ $(n=55)$ estavam com sobrepeso e $31,2 \%$ ( $n=39)$ obesos, evidenciando que $75,2 \%$ $(n=94)$ estavam acima do peso recomendado à sua compleição física. Outros estudos encontraram $53 \%$ e $52,5 \%$ dos participantes com sobrepeso ou obesidade (COSTA ET AL., 2007; SARNO; MONTEIRO, 2007).

No que concerne à QV, ainda são incipientes os estudos que utilizam o Minichal-Brasil, todavia, pode-se evidenciar que a média da QV foi de 67,82 numa escala de 0 a 100, pouco abaixo da média encontrada no estudo de validade interna do instrumento. Os domínios, 'psicológico e manifestações somáticas', apresentaram médias respectivamente de 88,01 e 91,84. Em estudo que utilizou o Minichal-Brasil, a média da QV, foi de 69,7. As médias por domínios foram 'estado mental' de 69,1 e no domínio 'manifestações somáticas' de 69,9 (MELCHIORS ET AL., 2010).

A análise de dependência entre as variáveis evidenciou diferença estatisticamente significativa entre QV e raça/cor $(\mathrm{p}=0.0002)$. A maior deterioração da QV tem sido apontada entre os adolescentes pertencentes às minorias étnicas. Um estudo demonstrou que a auto-avaliação da saúde foi melhor entre os adolescentes da raça branca $(\mathrm{p}<0,05)$ (HUANG, 2004).

Por fim, a variável escolaridade, também apresentou diferença estatisticamente significativa $(\mathrm{p}=0.001)$. Diferença similar foi encontrada (ALEXANDRE; CORDEIRO; RAMOS, 2008) utilizando, todavia um instrumento diferente para medida da $\mathrm{QV}$, sendo que os não alfabetizados apresentaram menores médias em todos os domínios.

Foi possível evidenciar que a QV dos informantes apresenta níveis considerados normais de acordo com a literatura vigente. Ademais, o teste de hipótese mostrou que mesmo entre os grupos que apresentavam níveis elevados de PA e normais no momento do exame, não houve diferença. Há a necessidade de acompanhamento e novos estudos para confirmar os achados, entretanto os resultados deste estudo podem evidenciar que o convívio com a HAS não é, isoladamente, um fator depressor da QV.

\section{Conclusão}

O estilo de vida dos informantes é composto, predominantemente, por bons hábitos de vida, como o fato de em sua maioria não 
serem fumantes e nem usuários de álcool, sem história do uso de cigarro ou álcool. Todavia foi possível identificar que a grande maioria não possui o hábito de praticar atividade física regular, considerado de suma importância para o controle da HAS. O perfil comportamental da amostra justifica os bons níveis de QV apresentados pela amostra, todavia, alguns fatores que influenciam na mesma puderam ser identificados.

Os aspectos da QV avaliados mostraram a existência de correlação entre os aspectos somáticos e os mentais. Este fato mostra que a já fadada ligação entre a saúde mental e corporal encontra-se presentes na população estudada, sendo um dos potenciais fatores depressores da saúde física dos indivíduos por consequência do acometimento da saúde mental e também da depreciação da QV.

Quanto aos demais fatores que influenciam na QV pôde-se identificar que a escolaridade e a raça/cor dos entrevistados influenciam de forma acentuada nesta quantificação, sendo que 52 \% dos que foram classificados com boa QV eram alfabetizados, e 45,6\% dos avaliados como baixa QV eram da categoria não brancos.

\section{Referências}

ALEXANDRE, T. S.; CORDEIRO, R. C.; RAMOS, L. R. Fatores associados à qualidade de vida em idosos com osteoartrite de joelho. Fisioterapia e Pesquisa, São Paulo, v. 15, n. 4, p. 326-332, dez. 2008.

BLOCH, K. V.; MELO, A. N.; NOGUEIRA, A. R. Prevalência da adesão ao tratamento anti-hipertensivo em hipertensos resistentes e validação de três métodos indiretos de avaliação da adesão. Cadernos de Saúde Pública, Rio de Janeiro, v. 24, n. 12, p. 2979-2984, 2008.

BRASIL. Ministério da Saúde. Secretaria de Gestão Estratégica e Participativa. VIGITEL BRASIL 2010: Vigilância de fatores de risco e proteção para doenças crônicas por inquérito telefônico. Brasília, DF: Ministério da Saúde, 2011. 152 p.

BRITO, D. M. S. et al. Qualidade de vida e percepção da doença entre portadores de hipertensão arterial Quality of life and perception of illness among individuals with high blood pressure. Caderno de Saúde Pública, Rio de Janeiro v. 24, n. 4, p. 933-940, 2008.

CIPULLO, J. P. et al. Prevalência e fatores de risco para hipertensão em uma população urbana brasileira. Arquivos Brasileiros de Cardiologia, Rio de Janeiro, v. 94, n. 4, p. 519-526, abr. 2010.

CONCEIÇÃO, T. V. et al. Valores de pressão arterial e suas associações com fatores de risco cardiovasculares em servidores da Universidade de Brasília. Arquivos
Brasileiros de Cardiologia, Rio de Janeiro, v. 86, n. 1, p. 26-31, jan. 2006.

CORSO, A. N. et al. Impacto de sintomas depressivos na qualidade de vida de usuários da rede básica de saúde. Revista Brasileira de Medicina, São Paulo, v. 30, n. 2, p. 257-262, 2009.

COSTA, J. S. D. et al. Prevalência de hipertensão arterial em adultos e fatores associados: um estudo de base populacional urbana em Pelotas, Rio Grande do Sul, Brasil. Arquivos Brasileiros de Cardiologia, Rio de Janeiro, v. 88, n. 1, p. 59-65, jan. 2007.

HELENA, E. T. S.; NEMES, M. I. B.; ELUF-NETO, J. Avaliação da assistência a pessoas com hipertensão arterial em Unidades de Estratégia Saúde da Família. Saúde e Sociedade, São Paulo, v. 19, n. 3, p. 614-626, set. 2010.

HUANG, G. H. Self-rated Health among Young People with Type 1 Diabetes in Relation to Risk Factors in a Longitudinal Study. American Journal of Epidemiology, Oxford, v. 159, n. 4, p. 364-372, 15 fev. 2004.

JESUS, S. et al. Perfil de um grupo de hipertensos : aspectos biossociais, conhecimentos e adesão ao tratamento. Acta Paulista de Enfermagem, São Paulo, v. 21, n. 1, p. 59-65, 2008.

KEARNEY, P. M. et al. Global burden of hypertension: analysis of worldwide data. Lancet, London v. 365, n. 
9455, p. 217-223, 2005.

LEMOS, S. S. Fatores que interferem na qualidade de vida com diabetes : a opinião dos portadores. Brasília, DF: Universidade de Brasília, 2008.

LIMA, H. P. et al. Adesão do usuário hipertenso ao tratamento e a interface com o saber sobre o agravo. Revista da Rede de Enfermagem do Nordeste, Fortaleza, v. 11, n. 2, p. 170-178, 2010.

MATOS, A. C.; LADEIA, A. M. Avaliação de Fatores de Risco Cardiovascular em uma Comunidade Rural da Bahia. Arquivos Brasileiros de Cardiologia, Rio de Janeiro, v. 81, n. 3, p. 291-296, 2003.

MELCHIORS, A. C. et al. Qualidade de vida em pacientes hipertensos e validade concorrente do MinichalBrasil. Arquivos Brasileiros de Cardiologia, Rio de Janeiro, v. 94, n. 3, p. 357-364, mar. 2010.

MINAYO, M. C. S.; HARTZ, Z. M. A.; BUSS, P. M. Qualidade de vida e saúde: um debate necessário. Ciência \&t Saúde Coletiva, Rio de Janeiro, v. 5, n. 1, p. 7-18, 2000

OLIVEIRA, A. F. C.; NOGUEIRA, M. S. Obesidade como fator de risco para a hipertensão entre profissionais de enfermagem de uma instituição filantrópica. Revista da Escola de Enfermagem da USP, São Paulo, v. 44, n. 2, p. 388-394, jun. 2010.

PASSOS, V. M. A.; ASSIS, T. D.; BARRETO, S. M. Hipertensão arterial no Brasil: estimativa de prevalência a partir de estudos de base populacional. Epidemiologia e Serviços de Saúde, Brasília, DF, v. 15, n. 1, p. $35-45$, mar. 2006
PESSUTO, J.; CARVALHO, E. C. Fatores de risco em indivíduos com Hipertensão Arterial. Revista LatinoAmericana de Enfermagem, Ribeirão Preto, v. 6, n. 1, p. 33-39, 1998

RABELO, D. F. et al. Qualidade de vida, condições e autopercepçãoda saúde entre idosos hipertensos e não hipertensos. Revista Kairós Gerontologia, São Paulo, v. 13, n. 2, p. 115-130, mar. 2011.

SANTA-HELENA, E. T.; NEMES, M. I. B.; ELUF-NETO, J. E. Fatores associados à não-adesão ao tratamento com anti-hipertensivos em pessoas atendidas em unidades de saúde da família. Caderno de Saúde Pública, Rio de Janeiro, v. 26, n. 12, p. 2389-2398, 2010.

SARNO, F.; MONTEIRO, C. A. Importância relativa do Índice de Massa Corporal e da circunferência abdominal na predição da hipertensão arterial. Revista de Saúde Pública, São Paulo, v. 41, n. 5, p. 788-796, out. 2007

SCHULZ, R. B. et al. Validação do mini-questionário de qualidade de vida em hipertensão arterial (MINICHAL) para o português (Brasil). Arquivos Brasileiros de Cardiologia, Rio de Janeiro, v. 90, n. 2, p. 139-144, fev. 2008.

VI DIRETRIZES BRASILEIRAS DE HIPERTENSÃO ARTERIAL. Revista Hipertensão, Rio de Janeiro, v. 13, n. 1, p. 1-13, 2010.

\footnotetext{
Recebido para publicação em abril de 2014

Versão final em novembro de 2014

Conflito de interesse: inexistente

Suporte financeiro: não houve
} 